\title{
The effect of viscosity, friction, and sonication on the morphology and metabolite production from Aspergillus terreus ATCC 20542
}

\begin{abstract}
This study investigates the effects of viscosity, friction, and sonication on the morphology and the production of lovastatin, (+)-geodin, and sulochrin by Aspergillus terreus ATCC 20542. Sodium alginate and gelatine were used to protect the fungal pellet from mechanical force by increasing the media viscosity. Sodium alginate stimulated the production of lovastatin by up to $329.0 \%$ and sulochrin by $128.7 \%$, with inhibitory effect on (+)-geodin production at all concentrations used. However, the use of gelatine to increase viscosity significantly suppressed lovastatin, (+)-geodin, and sulochrin's production (maximum reduction at day 9 of $42.7,60.8$, and $68.3 \%$, respectively), which indicated that the types of chemical play a major role in metabolite production. Higher viscosity increased both pellet biomass and size in all conditions. Friction significantly increased (+)-geodin's titre by $1527.5 \%$, lovastatin by $511.1 \%$, and sulochrin by $784.4 \%$ while reducing pellet biomass and size. Conversely, sonication produced disperse filamentous morphology with significantly lower metabolites. Sodium alginate-induced lovastatin and sulochrin production suggest that these metabolites are not affected by viscosity; rather, their production is affected by the specific action of certain chemicals. In contrast, low viscosity adversely affected (+)-geodin's production, while pellet disintegration can cause a significant production of $(+)$-geodin.
\end{abstract}

Keyword: Aspergillus terreus; Morphology; Lovastatin; (+)-Geodin; Sulochrin 